\title{
Megahertz all-optical swept-source optical coherence tomography based on broadband amplified optical time-stretch
}

\author{
Jingjiang Xu, Chi Zhang, Jianbing Xu, K. K. Y. Wong, and K. K. Tsia* \\ Department of Electrical and Electronic Engineering, The University of Hong Kong, Pokfulam Road, Hong Kong, China \\ ${ }^{*}$ Corresponding author: tsia@hku.hk
}

Received October 24, 2013; revised December 17, 2013; accepted December 23, 2013;

posted December 23, 2013 (Doc. ID 200097); published January 28, 2014

\begin{abstract}
We demonstrate all-optical ultrahigh-speed swept-source optical coherence tomography (OCT) based on amplified optical time-stretch (AOT). Such an inertia-free wavelength-swept mechanism, via group velocity dispersion, enables us to realize OCT with an A-scan rate well above MHz. More importantly, the key significance of AOT-OCT is its simultaneous broadband Raman amplification during the time-stretch process-greatly enhancing the detection sensitivity compared with prior attempts to apply optical time-stretch to OCT. Here, we report on an AOT-OCT system which is operated at an A-scan rate of $7.14 \mathrm{MHz}$, a superior roll-off performance ( $>2 \mathrm{~mm} / \mathrm{dB})$, a record-high sensitivity of time-stretch-based OCT ( $>80 \mathrm{~dB}$ ) with a broadband gain bandwidth of $80 \mathrm{~nm}$, which results in an axial resolution of $\sim 15 \mu \mathrm{m}$. Our AOT-OCT system is thus able to, for the first time to the best of our knowledge, perform time-stretch-based OCT of biological tissue in vivo. It represents a major step forward in utilizing AOT as an alternative for achieving practical MHz OCT, without any long-term mechanical stability concerns as in typical sweptsource OCT or bypassing the speed limitation of the image sensor employed in spectral-domain OCT. (C) 2014 Optical Society of America

OCIS codes: (110.4500) Optical coherence tomography; (170.4500) Optical coherence tomography; (170.3880) Medical and biological imaging; (190.5650) Raman effect. http://dx.doi.org/10.1364/OL.39.000622
\end{abstract}

Among all optical bioimaging modalities, optical coherence tomography (OCT) has been leading the way in terms of its acceptance in clinical applications, primarily due to its unique capability of microscopic label-free visualization and assessment of tissue structure in three dimensions (3D) without the need for excisional biopsy and histopathology [1]. In many OCT-based clinical diagnoses or image-guided intra-operative surgeries, there is the need for volumetric imaging in real-time. The key premise of real-time or even video-rate 3D OCT is an ultrafast A-scan rate of $>\mathrm{MHz}$. Most of the commercialgrade OCT systems, which are based on Fourier-domain techniques running at one to tens of $\mathrm{kHz}$, are still orders of magnitude slower than the $\mathrm{MHz}$ regime. Speed scaling has thus been one of the most investigated areas in OCT.

Fourier-domain OCT, categorized in two main classes as spectral-domain OCT (SD-OCT) and swept-source OCT (SS-OCT), has been the most viable approach to advance the A-scan rate. For example, SD-OCT at $\sim 100-200 \mathrm{kHz}$ has been demonstrated based on highspeed line-scan cameras [2, 3 ] . SS-OCT has also been shown to reach hundreds of $\mathrm{kHz}$ based on Fourierdomain mode-locked (FDML) lasers or swept-sources using rapidly rotating polygonal mirrors $[\underline{4}, \underline{5}]$. However, $\mathrm{MHz}$ OCT remains largely untapped because of the lack of $\mathrm{MHz}$ camera technology, as well as the intrinsic speed limitation imposed by the mechanical moving parts in the swept sources, e.g., piezoelectric-driven Fabry-Pérot cavities or rotating-mirrors $(\sim 100 \mathrm{kHz})$. Until recently, a combination of techniques, such as time-multiplexing of multiple wavelength-swept waveforms and multispot illumination, have been incorporated in SS-OCT and enabled $\mathrm{MHz}$ operation [6,7]. It has also been shown possible to achieve $1 \mathrm{MHz}$ SS-OCT based on microelectromechanical system (MEMS) []]. As far as long-term mechanical stability is concerne $\bar{d}$, it would be of great value to exploit an inertia-free mechanism for implementing an ultrafast swept-source.

An all-optical swept-source based on optical timestretch represents a potential alternative for realizing practical $\mathrm{MHz}$ OCT. Optical time-stretch is a process which maps the wavelength spectrum of a broadband optical pulse into time via group velocity dispersion (GVD) in a dispersive medium, mostly optical fibers. It has been successfully applied to ultrafast spectroscopy [9], microscopy [10], and all-optical digitization [11]. Adopting optical time-stretch in OCT has a threefold benefit

(i) The broadband pulse is optically wavelength-swept (or time-stretched) by GVD in a dispersive fiber (e.g., dispersion compensated fiber (DCF), or chirped fiber Bragg grating [12]), without the need for mechanical wavelength tuning.

(ii) The A-scan rate is governed by the repetition rate of the laser pulse train, which is typically tens of $\mathrm{MHz}$ for most of the broadband pulse lasers.

(iii) More importantly, broadband optical amplification can be implemented simultaneously to compensate the trade-off between optical loss and GVD in the dispersive fiber and thus greatly enhances the sensitivity of OCT [13].

To date, time-stretch-based OCT is yet to be proven promising for bioimaging. It is primarily because previous work had either limited source bandwidth $(\sim 10 \mathrm{~nm})$ which yields insufficient axial resolution of OCT $[14,15]$ or lack of broadband optical amplification which degrades the sensitivity $(<40 \mathrm{~dB})$ [16]. In this Letter, we demonstrate, for the first time to the best of our knowledge, $\mathrm{MHz}$ OCT of biological tissue using timestretch OCT. Specifically, we demonstrate broadband amplification $(>80 \mathrm{~nm})$ in the time-stretch process enabling a sensitivity as high as $82 \mathrm{~dB}$ which is comparable to 
conventional SS-OCT. We term our technique as amplified time-stretch OCT (AOT-OCT).

The experimental setup is shown in Fig. 1. A modelocked fiber laser (MLFL) (pulse width $=\overline{5} .8 \mathrm{ps}$ and center wavelength $=1554.8 \mathrm{~nm}$ ) is pulse-picked by an amplitude modulator (AM) in order to attain a repetition rate to $7.14 \mathrm{MHz}$ which ensures an $\sim 100 \%$ duty cycle of the swept waveform after the AOT process. After the erbium-doped fiber amplifier (EDFA) and an optical band pass filter (OBPF), the pulse is amplified to achieve a peak power of $\sim 200 \mathrm{~W}$ and is used to generate a supercontinuum (SC) through a $50 \mathrm{~m}$ highly nonlinear fiber $(\mathrm{HNLF})$ (zero-dispersion wavelength $=1554 \mathrm{~nm}$ ). After SC generation, we use a wavelength-division multiplexer (WDM) to select a spectral range from 1570 to $1700 \mathrm{~nm}$ for AOT-OCT. The broadband pulses are then timestretched by a $10.6 \mathrm{~km}$ DCF with a total GVD of $\sim-1 \mathrm{~ns} / \mathrm{nm}$. Simultaneous with the time-stretch process, we implement broadband-distributed Raman amplification within the same DCF. This step is critical to compensate for the inherent dispersive loss and provides optical gain to enhance the sensitivity for AOT-OCT. To implement broadband Raman amplification, we employ two co-propagating pump lasers at 1490 and $1509 \mathrm{~nm}$ (FOL1405RTD-657-1509, FOL1425RUX-617-1490, Fitel) and another two counter-propagating pump lasers at 1535 and $1561 \mathrm{~nm}$ (Tunable Laser $3646 \mathrm{RA}$ 00, TunicsOM amplified by the EDFA). The output powers of the pump lasers are 150-300 $\mathrm{mW}$. After the AOT process and another stage of a booster optical amplifier (BOA108000TSSAA, Covega), a train of ultrafast wavelength-swept waveforms at a repetition rate of $7.14 \mathrm{MHz}$ (average power of $\sim 15 \mathrm{~mW}$ ) is generated. This swept-source is generated all-optical by AOT and is employed for AOTOCT. The two-dimensional (2D) image is acquired by scanning the laser in the transverse direction using a galvanometric mirror. Finally we utilize a high- speed photodetector $(15 \mathrm{GHz})$ and a real-time oscilloscope ( $80 \mathrm{bGS} / \mathrm{s})$ to capture the interferograms from the AOT-OCT setup.

The AOT wavelength-swept waveform captured by the real-time oscilloscope shows a good agreement with the spectrum captured by an optical spectrum analyzer

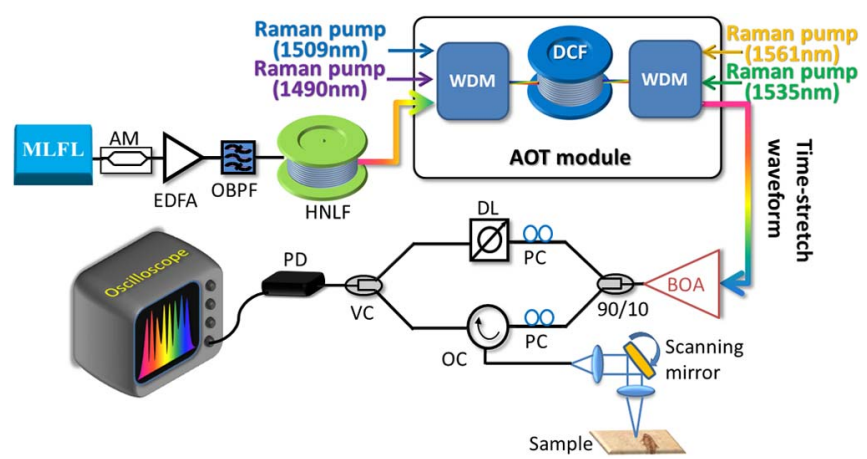

Fig. 1. Schematic of the AOT-OCT. MLFL, mode-locked fiber laser; PC, polarization controller; AM, amplitude modulator; EDFA, erbium-doped fiber amplifier; OBPF, optical band pass filter; HNLF, highly nonlinear fiber; WDM, wavelength-division multiplexer; DCF, dispersion compensation fiber; BOA, booster optical amplifier; DL, delay line; OC, optical circulator; VC, variable coupler; $\mathrm{PD}$, photodetector.
(OSA), confirming the wavelength-time mapping in AOT [Fig. 2(a)]. We note that the nonlinear wavelength-time mapping due to higher order GVD becomes more obvious for broadband AOT wavelength-swept waveform [a full width at half-maximum (FWHM) of $\sim 80 \mathrm{~nm}$ in our case] and thus has to be taken into account in the wavelength calibration for AOT-OCT. We calibrate such nonlinear wavelength-time mapping by retrieving the wavelengthdependent dispersion curve of the DCF [Fig. 2(b)] by a phase-retrieval algorithm based on a Hilbert transform of the raw wavelength-swept waveform [Fig. 2(a)].

Figure 3(a) shows the time-stretch interferograms captured by the real-time oscilloscope. With an average GVD of $\sim-1 \mathrm{~ns} / \mathrm{nm}$ [see Fig. 2(b)], the full-swept range of $120 \mathrm{~nm}(1570-1690 \mathrm{~nm})$ is mapped into a time span of $\sim 120$ ns, corresponding to an $\sim 100 \%$ duty cycle. We use a silver mirror in the sample arm to evaluate the singleshot point spread function (PSF), which is derived from the recorded interferograms. The measured axial resolution of the single-shot PSF is $15 \mu \mathrm{m}$ which agrees well with the theoretical calculation of $14 \mu \mathrm{m}$, assuming a Gaussian spectral shape with a FWHM of $80 \mathrm{~nm}$ centered at $1620 \mathrm{~nm}$. The sensitivity is measured with $\sim 10 \mathrm{~mW}$ on a mirror in the sample arm. By taking into account the insertion loss of the fiber components and interferometer losses, the sensitivity of the current system is $\sim 82 \mathrm{~dB}$, which is, to the best of our knowledge, the highest sensitivity among the prior time-stretch-based OCT demonstrations [14-16]. Such a high sensitivity in AOT-OCT is made possible by the broadband amplification contributed from both the distributed Raman amplification in the DCF (on-off gain of $\sim 15 \mathrm{~dB}$ and net gain of $\sim 5 \mathrm{~dB}$ ), as well as the BOA (net gain of $\sim 15 \mathrm{~dB}$ ) [Fig. 3(b)]. The two-stage amplification is essential not only for enhancing the gain bandwidth, as opposed to the single-stage [see Fig. 3(b)], it also improves the signal-to-noise ratio (SNR) and thus the sensitivity due to the lower overall noise figure in the system (estimated to be $\sim 6 \mathrm{~dB}[17]$ ) when both amplification stages are included, compared to the case of having only BOA (noise figure of $\sim 8 \mathrm{~dB}$ ). This can be shown in Fig. 3(c) that the SNR is improved from $\sim 35$ to $\sim 45 \mathrm{~dB}$ when Raman amplification is present before the BOA.

By varying the mirror position in the axial direction, we plot the roll-off curve of the AOT-OCT system (Fig. 4). The roll-off number achieved in our system is $2.2 \mathrm{~mm} / \mathrm{dB}$ up to the depth at $\sim 15 \mathrm{~mm}$ which is $\sim 20$ times better than that of FDML-OCT at a similar speed of $5.2 \mathrm{MHz}$ effective
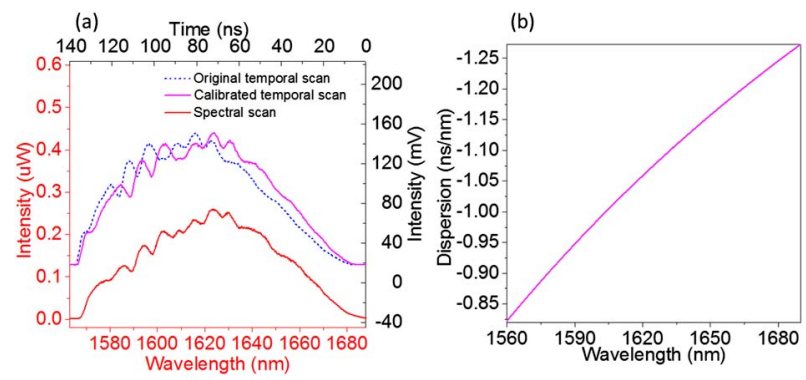

Fig. 2. (a) Raw spectra after the AOT is captured by an OSA (red line) and by a real-time oscilloscope (blue line). (b) The spectrum (magenta line) corresponds to the calibrated temporal scan by the dispersion curve. 
(a)
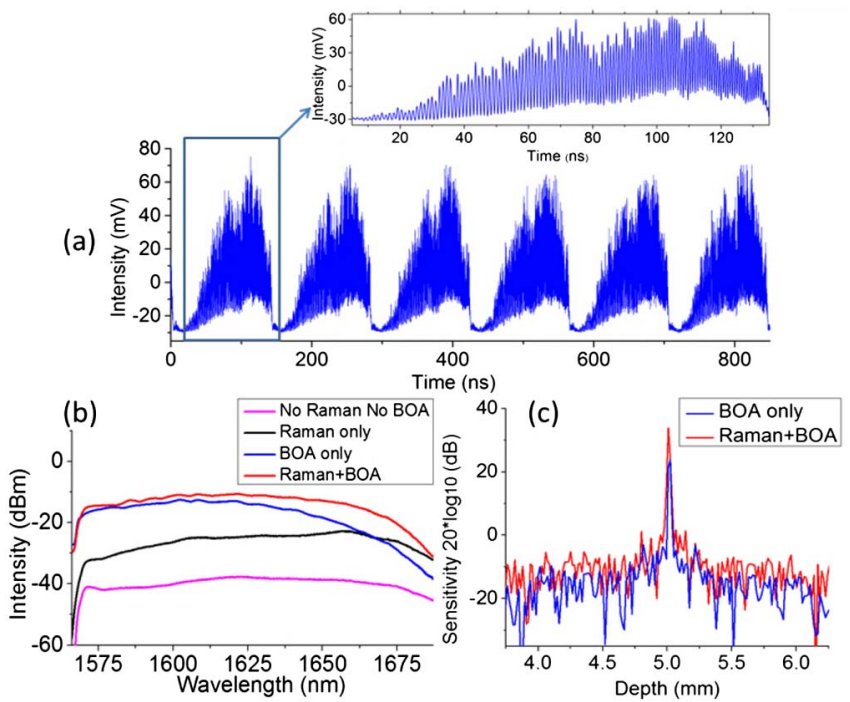

Fig. 3. (a) Time-stretch interferograms at a rate of $7.14 \mathrm{MHz}$ captured in real-time. The inset shows the zoom-in view of one period. (b) Time-stretch spectra with different amplification scenarios: (red line) with both Raman amplification and BOA, (blue line) with BOA only, (black line) with Raman amplification only, and (magenta line) without any amplification. (c) Measured PSFs with (red line) and without (blue line) Raman amplification before the BOA.

swept rate [6]. The calculated instantaneous linewidth is $132 \mathrm{pm}$, based on stationary-phase approximation in the AOT process experimental parameters [18]. It corresponds to an instantaneous coherence length as long as $\sim 1 \mathrm{~cm}$ which is consistent with the roll-off performance. The roll-off drops more significantly beyond $20 \mathrm{~mm}$ because of the characteristic frequency response of the photodetector with a bandwidth of $15 \mathrm{GHz}$. We note that the long depth range is enabled all-optical by the considerable amount of GVD (>ns/nm) which results in high spectral resolution, i.e., instantaneous linewidth [13]. This is a unique feature to AOT. In contrast to FDML, the coherence length is largely governed by the cavity linewidth of the tunable filter which is subject to mechanical instability. AOT-OCT is also not subject to the swept rates, as in the case of FDML. AOT-OCT system could thus particularly benefit the applications which require long-range imaging, i.e., full-eye imaging or endoscopic tissue imaging $[\underline{7}, \underline{19}]$.

In our AOT-OCT system, careful design of the multiplestage broadband amplification (both the gain and noise figure), i.e., broadband Raman amplification followed by

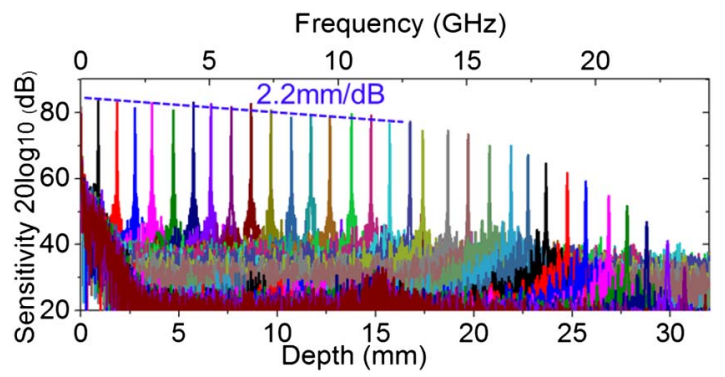

Fig. 4. Roll-off performance of the AOT-OCT. PSFs measured at different depths in air.
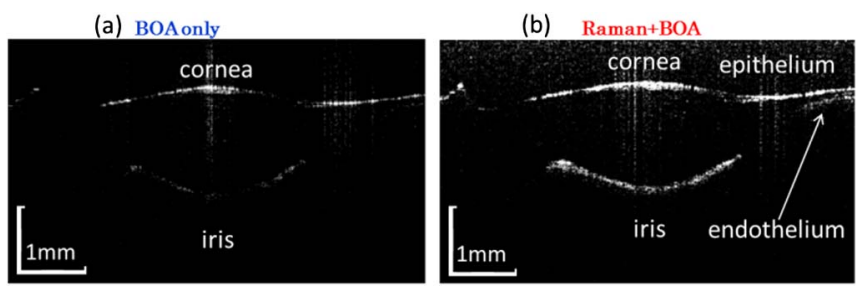

Fig. 5. Effect of the Raman amplification on the AOT-OCT image. (a) An image of the anterior segment of the fish eye with BOA only. (b) The same part of the fish eye with both Raman amplification and BOA.

BOA, plays an important role for improving image quality. The importance of Raman amplification before BOA in AOT-OCT can be clearly exemplified by the images of the anterior segment of a fish eye shown in Fig. 5. With the $10 \mathrm{~dB}$ improvement in sensitivity because of the twostage gain with better SNR, the two layers of corneal epithelium and endothelium as well as the iris are clearly visible [Fig. 5(b)]. This is in contrast to the case without the Raman amplification [Fig. 5(a)], in which only the rough outlines of the cornea and iris could be observed.

We could also take advantage of the ultrafast sweptrate operation to perform averaging of multiple A-scans in order to further improve image quality without compromising the speed significantly. By taking an average of six consecutive A-scans resulting in an effective frame rate of $1.2 \mathrm{MHz}$, we captured the AOT-OCT images of the anterior segment of the fish eye [Fig. 6(a)] and human finger print in vivo [Fig. 6(b)]. The data set is 500 (transverse) $\times 2000$ (axial) pixels with a 20 Gsamples $/ \mathrm{s}$ digitizer. Our system is able to reveal the epidermis and dermis layers, as well as the sweat ducts [Fig. 6(b)]. We note that this is the first demonstration of time-stretchbased OCT for imaging biological tissue in vivo with reasonable image quality. Notably, averaging six consecutive A-scans can alleviate the sensitivity deterioration by the intensity fluctuation of the SC source and thus results in further improvement of the sensitivity up to $\sim 90 \mathrm{~dB}$ at the scan rate of $1.2 \mathrm{MHz}$.

The sensitivity of the current AOT-OCT system is lower than the theoretical shot-noise limit of a swept-source OCT system operating at the same $7.14 \mathrm{MHz}$, which is

(a)

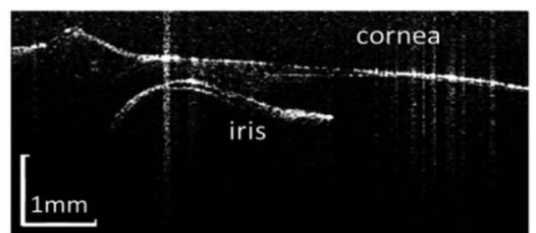

(b)

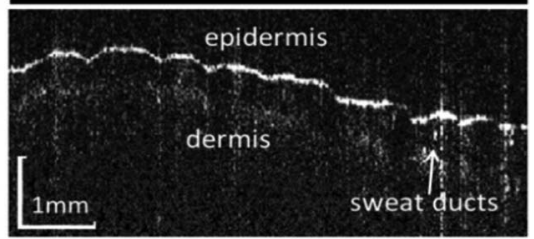

Fig. 6. AOT-OCT images of (a) the anterior segment of the fish eye and (b) a human finger print. represents a major step forward in utilizing AOT as an attractive alternative for achieving practical $\mathrm{MHz}$ OCT. 
$98 \mathrm{~dB}$ according to [7]. Such deviation can be attributed to

(i) The insertion loss of the setup, including free-space coupling loss or fiber component loss.

(ii) The spectral fluctuation of the broadband source, i.e., the picosecond (ps) SC source. This can be observed from the evaluated noise level within the depth range up to $\sim 3 \mathrm{~mm}$ in the roll-off plot (Fig. 4). It is primarily due to the noise-driven dynamics (or more specifically, modulation instability) in the long-pulse (>ps) SC generation, especially when the SC is pumped in the anomalous dispersion regime [20]. For this reason, the zero-delay position of the AOT-OCT images here is set at $3 \mathrm{~mm}$. The stability can be improved by utilizing a femtosecond SC [20], seeded SC [21], or normal dispersion pumping scheme [22]. It also can be improved by averaging the A-scans as discussed earlier.

(iii) The AOT-OCT system is in practice not shot-noise limited because of the amplifier noise, careful design of multiple-stage amplifier is essential (e.g., optimizing the cascaded amplifiers' gain and noise figures as well as their positions in the cascaded system [17]) in order to minimize the overall noise figure, which affects the sensitivity of AOT-OCT.

While the current AOT-OCT system presented here is operated in $\sim 1600 \mathrm{~nm}$ wavelength range, AOT-OCT can in principle be operated in the shorter wavelength windows ( 1300 or $1060 \mathrm{~nm}$ ) because the dispersive fibers with reasonably high GVD $(\sim \mathrm{ns} / \mathrm{nm})$ and low loss $(<1 \mathrm{~dB} / \mathrm{km})$ are commercially available for these wavelength ranges. Also, Raman amplification has the advantages of its widely tunable gain spectra and the flexible gain spectral shaping by dispersion engineering of the fibers or multiple-pumping schemes. As a result, AOT-OCT could be implemented in different wavelength regimes and thus could benefit a wide range of OCT applications. Last but not least, the current 2D imaging speed is mainly limited by the off-line processing speed. High-speed realtime imaging processing can be implemented by the high-performance computing processors, e.g., a fieldprogrammable gate array or a graphics processor unit [23].

In conclusion, we demonstrated a high-speed alloptical MHz SS-OCT modality AOT-OCT. Implementing broadband amplification simultaneously with timestretch, the AOT-OCT demonstrated here achieved a record high sensitivity of above $80 \mathrm{~dB}$ at an A-scan rate of $7.14 \mathrm{MHz}$, compared with all the prior time-stretch-based OCT. The superior roll-off performance $(>2 \mathrm{~mm} / d B)$ together with its broadband gain $(\sim 80 \mathrm{~nm})$, which results in an axial resolution of $15 \mu \mathrm{m}$, enables the first demonstration, to the best of our knowledge, of using timestretch for in vivo OCT of biological tissue. Without any mechanical stability concern (as in SS-OCT) or speed limitation of the image sensor technology (as in SDOCT), the present AOT-OCT demonstration.

The work in this Letter is partially supported by grants from the Research Grants Council of Hong Kong Special Administrative Region, China (HKU 717212E, HKU $717510 \mathrm{E}, \mathrm{HKU} 717911 \mathrm{E}$, and HKU 720112E), and the University Development Fund of HKU.

\section{References}

1. W. Drexler and J. G. Fujimoto, Optical Coherence Tomography: Technology and Applications (Biological and Medical Physics, Biomedical Engineering) (Springer, 2008).

2. H. C. Lee, J. J. Liu, Y. Sheikine, A. D. Aguirre, J. L. Connolly, and J. G. Fujimoto, Biomed. Opt. Express 4, 1236 (2013).

3. L. An, P. Li, G. P. Lan, D. Malchow, and R. K. K. Wang, Biomed. Opt. Express 4, 245 (2013).

4. R. Huber, M. Wojtkowski, and J. G. Fujimoto, Opt. Express 14, 3225 (2006).

5. W. Y. Oh, S. H. Yun, G. J. Tearney, and B. E. Bouma, Opt. Lett. 30, 3159 (2005).

6. W. Wieser, B. R. Biedermann, T. Klein, C. M. Eigenwillig, and R. Huber, Opt. Express 18, 14685 (2010).

7. T. Klein, W. Wieser, L. Reznicek, A. Neubauer, A. Kampik, and R. Huber, Biomed. Opt. Express 4, 1890 (2013).

8. T. H. Tsai, B. Potsaid, Y. K. Tao, V. Jayaraman, J. Jiang, P. J. S. Heim, M. F. Kraus, C. Zhou, J. Hornegger, H. Mashimo, A. E. Cable, and J. G. Fujimoto, Biomed. Opt. Express 4, 1119 (2013).

9. D. R. Solli, J. Chou, and B. Jalali, Nat. Photonics 2, 48 (2008).

10. K. Goda, K. K. Tsia, and B. Jalali, Nature 458, 1145 (2009).

11. J. Chou, O. Boyraz, D. Solli, and B. Jalali, Appl. Phys. Lett. 91, 161105 (2007).

12. Y. Park, T. J. Ahn, J. C. Kieffer, and J. Azana, Opt. Express 15, 4597 (2007)

13. K. Goda, D. R. Solli, K. K. Tsia, and B. Jalali, Phys. Rev. A 80, 043821 (2009).

14. K. Goda, D. R. Solli, and B. Jalali, Appl. Phys. Lett. 93, 031106 (2008).

15. K. Goda, A. Fard, O. Malik, G. Fu, A. Quach, and B. Jalali, Opt. Express 20, 19612 (2012).

16. S. Moon and D. Y. Kim, Opt. Express 14, 11575 (2006).

17. C. Headley and G. Agrawal, Raman Amplification in Fiber Optical Communication Systems (Elsevier Academic, 2005).

18. K. K. Tsia, K. Goda, D. Capewell, and B. Jalali, Opt. Express 18, 10016 (2010).

19. I. Grulkowski, J. J. Liu, B. Potsaid, V. Jayaraman, J. Jiang, J. G. Fujimoto, and A. E. Cable, Opt. Lett. 38, 673 (2013).

20. J. M. Dudley, G. Genty, and S. Coen, Rev. Mod. Phys. 78, 1135 (2006).

21. K. K. Y. Cheung, C. Zhang, Y. Zhou, K. K. Y. Wong, and K. K. Tsia, Opt. Lett. 36, 160 (2011).

22. M. Nakazawa, K. Tamura, H. Kubota, and E. Yoshida, Opt. Fiber Technol. 4, 215 (1998).

23. A. E. Desjardins, B. J. Vakoc, M. J. Suter, S. H. Yun, G. J. Tearney, and B. E. Bouma, IEEE Trans. Med. Imaging 28, 1468 (2009). 